\title{
Interference of heavy-atom with magnetic spin effects in spin-correlated micellar radical pairs*
}

\author{
By JIE QIANG WU†, DIETRICH BAUMANN and ULRICH E. STEINER \\ Fakultät für Chemie, Universität Konstanz, D-78434 Konstanz, Germany
}

\begin{abstract}
The recombination kinetics of the radical pairs ${ }^{3}\left(\mathrm{SeH}^{*} . . \mathrm{An}^{*+}\right)$, with $\mathrm{SeH}^{+}=$selenine, $\mathrm{An}=$ aniline, and ${ }^{3}\left(\mathrm{TH}^{\cdot} . . \mathrm{An}^{*}\right)$ with $\mathrm{TH}^{+}=$thionine, $\mathrm{p}-\mathrm{Cl}-$ $\mathrm{An}=4$-chloroaniline, in benzene/cetyldimethylbenzylammonium chloride $/ \mathrm{H}_{2} \mathrm{O}$ reverse microemulsions was measured by ns-laser flash photolysis as a function of magnetic field and nanodroplet size. By comparison with the behaviour of the ${ }^{3}\left(\mathrm{TH}^{*} . . \mathrm{An}^{*+}\right)$ radical pairs evidence was provided that, besides the main route of triplet radical pair recombination through triplet to singlet spin conversion in situations when the radicals are separate and subsequent reaction on re-encountering as singlet radical pair, there is a finite probability that radical pairs encountering with overall triplet spin will directly recombine through electron transfer to singlet ground state products. The probability of this process, which is induced by spin-orbit coupling and is independent of a magnetic field, was evaluated for the three radical pairs specified above.
\end{abstract}

\section{Introduction}

Spin chemistry, i.e. magnetic field and spin effects observed on chemical reaction rates and yields, and chemically induced dynamic effects in magnetic resonance spectroscopy, have contributed a wealth of information on the kinetic behaviour and interactions in radical pairs (RPs) functioning as reaction intermediates in many chemical and photochemical reactions $[1-4]$. Due to the fact that radical recombination reactions are controlled by the overall spin multiplicity of the reacting RPs, electron spin evolution during the life-time of the RP becomes an essential constituent of the reaction mechanism and any factor controlling spin motion may affect the reactive behaviour of the $R P$, e.g. the ratio of products found in cage reactions against those of escape reactions. In the RP mechanism $[5,6]$ the role of isotropic hyperfine interaction as a means of controlling electron spin motion has been of particular interest because rather small external magnetic fields can efficiently interfere with coherent, hyperfine driven spin motion. Furthermore, this type of interaction also opens up the intriguing possibility of chemical isotope enrichment by exploiting the magnetic isotope effect $[7,8]$. The feasibility of such methods requires that other hyperfine independent mechanisms that may affect the RP spin should be of minor importance. Among such adverse mechanisms spin-orbit coupling (SOC) dependent ones are most important. In this article we shall address some questions related to the role of SOC in the spin chemistry of RPs.

Our investigations were carried out in micellar systems. Micellar environments have figured prominently in spin chemistry since Turro and Weed [9] first reported

* Dedicated to Professor Richard R. Schmidt on the occasion of his 60th birthday.

† Present address: Physikalisch-Chemisches Institut der Universität Zürich, Winterthurstrasse 190, 8057 Zürich, Switzerland. 
on the strong enhancement of such phenomena due to the micellar supercage effect. The supercage effect of micellar aggregates (these may be conceived as more or less closed nanoscopic reactors) greatly extends the time span during which spin correlated RPs may have geminate re-encounters and thereby the reaction of the re-encountering radicals will be able to sample more efficiently the spin evolution during the RP life-time. One important characteristic of the spin chemistry of RPs in micelles or other nanoscopic reactors is exhibited by the field dependence of the recombination kinetics, which usually is saturated at much higher fields than in low viscosity homogeneous solutions. This is a direct consequence of the long life-time of the correlated RP in the micelle which allows incoherent, $T_{1}$-type, spin transitions to contribute substantially to the spin evolution in the RP (relaxation mechanism $[10,11])$. The sign of the field effect in the field region governed by the relaxation mechanism usually is the same as at low fields where the hyperfine coupling (hfc) mechanism operates. The latter is inhibited more strongly than the former as the Zeeman splitting of the electron spin levels approaches and finally exceeds the characteristic hfc strength of the RP. The most important types of $T_{1}$-electron spin relaxation mechanisms exhibiting a decrease of the rate of spin relaxation $\left(1 / T_{1}\right)$ with increasing field are anisotropic hyperfine coupling (ahfc) and electron spin-spin dipolar interaction (sdi) [12]. Therefore, it is generally assumed that it is these two mechanisms which largely determine the field dependence of micellar RP kinetics in the field region between some $100 \mathrm{G} \dagger$ (where coherent hfc is usually suppressed) and about $1 \mathrm{~T}$, where the field effect tends to be saturated even in micellar solutions. Nevertheless, some kinetic activity of the outer Zeeman levels of a triplet RP in undergoing recombination to singlet products does persist even at fields higher than $1 \mathrm{~T}$. In principle, there are two possibilities to account for such a behaviour:

(i) One may invoke field-independent $T_{1}$-processes in the radicals which could become the dominating ones when the ahfc and sdi mechanisms are suppressed at high fields. Such mechanisms would be the $g$-tensor anisotropy mechanism (gta) (it becomes field independent at high fields) and the spin-rotational mechanism (sri), both related to intra-radical SOC effects.

(ii) A different possibility is the mechanism of intersystem crossing (ISC) occurring together with, i.e. kinetically inseparable from the recombination reaction, such that e.g. a RP encountering with triplet (T) spin reacts directly to products with singlet (S) spin. This mechanism, too, should be insensitive to a magnetic field (except for the rather extreme situation where the triplet mechanism can be invoked. [13-15]).

While possibility (ii) has been advocated as a mechanism of direct, 'spinforbidden' $\sigma$-bond formation in triplet biradicals [16-18] and triplet radical pairs [19] and has been invoked by Levin and Kuzmin [20] to analyse the magnetic field dependent kinetics of RP recombination in micelles, mechanism (i) has been considered a reasonable explanation for experimental results obtained in this laboratory [21] on the internal heavy-atom induced quenching of the magnetic field effect in the intramicellar recombination kinetics of T-RPs composed of semithionine and a series of halogen substituted aniline cation radicals. In the present paper we will show how the micelle-size dependence of the magnetic field effect (MFE) can be used to provide unequivocal experimental evidence that in the latter systems, too, it

$$
\dagger 1 \mathrm{G}=10^{-4} \mathrm{~T}
$$


is in fact mechanism (ii) which is responsible for the saturation value of the MFE. Furthermore, we shall demonstrate that this conclusion is true for heavy atom modification of either type of radical: electron donor or electron acceptor.

\section{General reaction scheme}

The general reaction scheme within which our experiments and pertinent theoretical problems will be discussed is given in scheme I. $T_{+}, T_{0}, T_{-}$and $S$ denote the respective spin substates of the intramicellar RP, which may decay due to escape $\left(k_{\text {esc }}\right)$ of one of the radicals into the bulk, or which may recombine to a singlet recombination product in a spin-allowed process $\left(k_{s}\right)$ from the singlet RP state or, a matter to be investigated, with direct reaction of a triplet RP to the singlet recombination product in a spin-forbidden process $\left(k_{T}\right)$. Apart from the chemical rate processes in which they are involved, the spin substates of the $R P$ can be interconverted by relaxation processes or by coherent coupling through isotropic hyperfine interaction. Although the details of the time dependence of the transitions induced coherently or incoherently are different (see [3]) both types of processes will be formally dealt with as first-order rate processes, an approximation justified with regard to the aspects considered in this investigation. Our notation for the rate constants is in line with that used by Hayashi and Nagakura [10]. If the RP is created with triplet spin it will decay, in general, in a biexponential process with rate constants given by $[10,12,20]$ :

$$
\begin{gathered}
k_{\mathrm{obs}(1,2)}=k_{\mathrm{esc}}+\frac{1}{4}\left(k_{S}+3 k_{T}\right)+k_{\mathrm{r}}+D_{1,2}, \\
D_{1,2}= \pm\left[k_{r}^{2}+\frac{1}{16}\left(k_{S}-k_{T}\right)^{2}\right]^{1 / 2} \\
k_{r}=k_{r, 1}+k_{r, 1}^{\prime} .
\end{gathered}
$$

In the derivation it has been assumed that

$$
k_{r, 0} \gg k_{\mathrm{S}}+k_{\mathrm{esc}} \geqslant k_{T}+k_{\mathrm{esc}},
$$

an assumption justified if isotropic hfe is strong enough. $\dagger$ At zero field, $k_{r, 1}=$ $k_{r, 1}^{\prime}=k_{r, 0}$. Therefore, because of inequality (4), in zero field $S / T$ spin equilibrium is

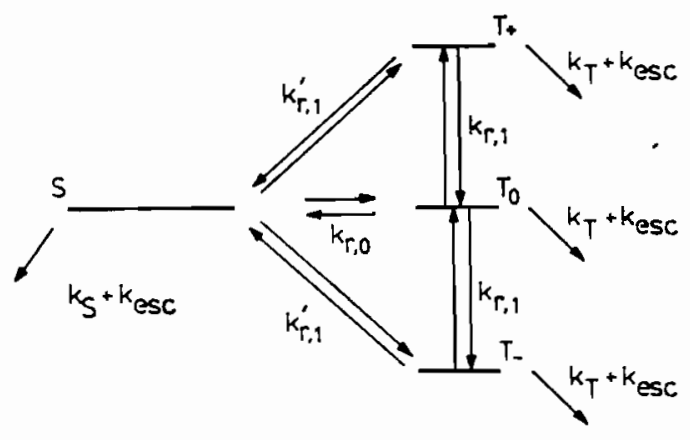

Scheme I

† The characteristic time constant of $T_{0} \rightleftarrows S$ equilibration in high field is [22] $\tau_{T_{0} S}=$ $\left[\frac{1}{6} \gamma_{\mathrm{e}} \sum a_{i}^{2} I_{i}\left(I_{i}+1\right)\right]^{-1 / 2} \equiv \sqrt{6}\left(\gamma_{\mathrm{e}} B_{\mathrm{eff}}\right)^{-1}$ where $\gamma_{\mathrm{e}}$ is the gyromagnetic ratio of the electron, $a_{i}$ the isotropic hfc constant of nucleus $i$ in Gauss, and $I_{i}$ its spin quantum number. For our RPs $B_{\text {eff }} \approx 20 \mathrm{G}$ yielding $\tau_{T_{0} s} \approx 7 \mathrm{~ns}$, a time less than one tenth of the typical re-encounter time in the micelles investigated here. 
established prior to and maintained during decay of the RP, while in high fields rapid spin equilibrium is only established between $T_{0}$ and $S$ spin substates. Experimentally, the rate constant of escape, $k_{\text {esc }}$, is easily separated from the observed overall kinetics by measuring the yield of escape radicals so that we may concentrate on the analysis of the rate constants of intramicellar recombination defined as $k_{\text {rec }} \equiv k_{\text {obs }}-k_{\text {esc }}$ and its magnetic field dependence $k_{\text {rec }}(B)$ resulting from the magnetic field dependence of $k_{r}$. The specific question that we want to address in this paper is: where will an increase of SOC, as arising from heavy atom substitution in the radicals involved, become apparent: in the enhancement of magnetic field independent relaxation processes (i.e. in $k_{r}$ ) or in the enhancement of the direct spin-forbidden RP recombination (i.e. in $k_{T}$ )?

\section{Experimental}

Thionine $\left(\mathrm{TH}^{+} \mathrm{Cl}^{-}\right)$from Janssen $(98 \%)$ was purified according to [23 a]. Selenine $\left(\mathrm{SeH}^{+} \mathrm{Cl}^{-}\right)$was obtained as a gift from Dr E. Vogelmann and Professor H. E. A. Kramer, Stuttgart. For synthesis and purification see [23 b]. Aniline (Merck, p.a.) was distilled in vacuum. 4-Chloroaniline (Fluka, $>99 \%$ ) and benzene (Merck, p.a.) were used as received. CDBA (cetyldimethylbenzylammonium chloride) from Fluka (97\%) was purified by treatment with active carbon and double fractional recrystallization from acetic acid ethyl ester. Water was deionized and doubly distilled. As usual, the water content in water/CDBA/benzene reverse micellar system has been characterized by the molar ratio of water to surfactant molecules, $w=\left[\mathrm{H}_{2} \mathrm{O}\right] /[\mathrm{CDBA}]$.

Isotropic, clear sample solutions were obtained by dissolving the appropriate amount of CDBA, water and aqueous stock solution of dye in benzene [24]. The total concentrations of dye and CDBA were $5 \times 10^{-6} \mathrm{M}$ and $0.04 \mathrm{M}$ respectively. For all experiments described here the electron donor compound was added immediately before the experiments. In order to avoid precipitations, the addition of the donor had to be carried out in small portions while stirring in the dark. Before investigation the solutions were flushed with a steady stream of $\mathrm{N}_{2}(>99.999 \%)$ for about $30 \mathrm{~min}$ in order to remove $\mathrm{O}_{2}$.

The laser flash spectrometer used for magnetic field dependent transient absorption measurements has been described in detail in [25]. An excimer-laser pumped dye laser tuned to $582 \mathrm{~nm}$ with rhodamine $6 \mathrm{G}$ as a laser dye was used. The laser pulse width was about $25 \mathrm{~ns}$. In the sample cuvette positioned between the pole pieces of a Bruker Model B-E15 electromagnet the probe light beam (from a pulsed Xenon arc lamp) crossed the exciting laser beam at a small angle. The cylindrical cuvette of $1 \mathrm{~cm}$ path-length and about $0.3 \mathrm{ml}$ volume was inserted in a flow system so that the contents of the cuvette was easily replaced after each laser shot. Typically, the time-resolved transient signals were averaged over a series of 32 laser pulses.<smiles>[Y]c1cc(N)ccc1N=C1C=CC(=N)C=C1</smiles>

$\mathrm{X}=\mathrm{S}:$ thionine $\left(\mathrm{TH}^{+}\right)$ $\mathrm{X}=\mathrm{Se}$ : selenine $\left(\mathrm{SeH}^{+}\right)$

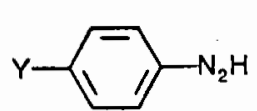

$\mathrm{Y}=\mathrm{H}:$ aniline (An)

$\mathrm{Y}=\mathrm{Cl}: \mathrm{p}-\mathrm{Cl}$-aniline (pCl-An) 


\section{Results and discussion}

General information on the kind of experiments carried out and analysed in this paper has been given in previous work from this laboratory (see $[21,26]$ ). The dye cations are solubilized within the water pools of water-in-oil microemulsions employing CDBA as a surfactant and benzene as the oil bulk phase. With the dye concentrations employed the probability that more than one dye molecule occupy the same microemulsion droplet is negligible. The radius of the nanometre-sized water droplets can be conveniently varied as a linear function of the molar ratio $(w)$ of water to surfactant with the following approximate relation for the radius of the 'water pools': $r_{\text {wp }}=1.61 w+4.28$, where $r_{\text {wp }}$ is given in $\AA$ units [12]. Upon laser flash excitation the excited triplet state of the dye is produced virtually instantaneously, i.e. synchronously with the flash. The excited dye triplet is quenched by electron transfer using some aniline derivative as an electron donor. Thereby isolated RPs with initial triplet spin correlation are produced, the decay kinetics of which is monitored on the nanosecond and microsecond time scale using laser flash transient absorption spectroscopy.

Our standard system for such investigations has been the RP consisting of semireduced thionine $\left(\mathrm{TH}^{*}\right)$ and aniline radical cation $\left(\mathrm{An}^{*}\right)$ obtained when reacting thionine triplet with aniline as electron donor. For comparison, examples of magnetic field dependent signals with this system are included in figure 1. The slowing down of the RP decay with the increase of magnetic field strength is clearly apparent. While the decay at non-zero magnetic field deviates from being monoexponential, the separability of the two exponentials is rather poor. Therefore to avoid ambiguity, all the decays were fitted by a monoexponential function,

$$
\frac{\left[\mathrm{TH}^{\bullet}\right]_{t}}{\left[\mathrm{TH}^{\bullet}\right]_{0}}=\left[\Phi_{\text {esc }}+\left(1-\Phi_{\mathrm{esc}}\right) \exp \left(-k_{\mathrm{obs}} t\right)\right] .
$$

From $\Phi_{\text {esc }}$ and $k_{\text {obs }}$, the recombination rate constant $k_{\text {rec }}$ is evaluated as

$$
k_{\text {rec }}=k_{\text {obs }}\left(1-\Phi_{\text {esc }}\right) \text {. }
$$

In our discussion we will consider this experimental $k_{\text {rec }}$ value as representing the behaviour of the slower decay component according to scheme 1 . While this must be certainly considered as an approximation it does not affect the validity of our conclusions.

\subsection{Effect of a heavy atom in the semireduced radical: selenine semiquinone}

SOC can be considerably increased by substituting the S-atom in thionine by Se. On the other hand, however, the spectroscopic properties and photochemical behaviour of selenine is very similar to that of thionine, as has been demonstrated with homogeneous solutions by Vogelman and Kramer [27]. For reverse micelles of the water/CDBA/benzene system the absorption spectra of selenine in the ground state $\left(\mathrm{SeH}^{+}\right)$, the semiquinone radical $\left({ }^{2} \mathrm{SeH}^{-}\right)$and the excited triplet state $\left({ }^{3} \mathrm{SeH}^{+}\right)$ are shown in figure 2 . The spectra in micellar solution are very similar to those reported in [27] for homogeneous solution and to the spectra of corresponding thionine species in the same micellar system [21]. The absorption maxima of the triplet ${ }^{3} \mathrm{SeH}^{+}, 430$ and $805 \mathrm{~nm}$, and the ground state $\mathrm{SeH}^{+}, 615 \mathrm{~nm}$, are shifted to the red by $5 \mathrm{~nm}$ as compared to thionine triplet ${ }^{3} \mathrm{TH}^{+}, 425$ and $800 \mathrm{~nm}$, and thionine 

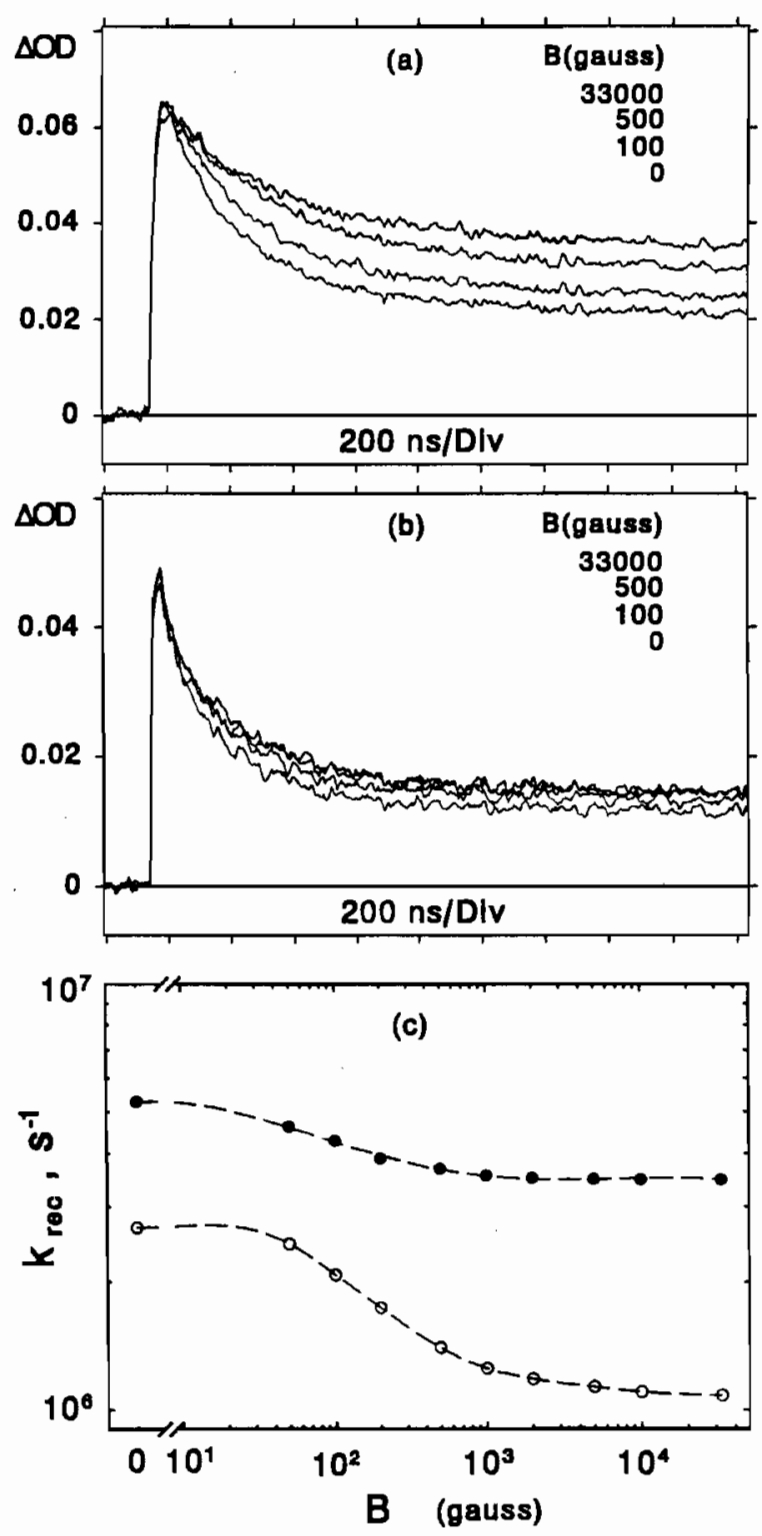

Figure 1. (a), (b) Transient absorption decay kinetics of T-RPs in water/CDBA/benzene reverse micellar solution $(w=15)$, monitored at the absorption maximum $(425 \mathrm{~nm})$ of the dye radicals for various magnetic field values with $(a){ }^{3}\left(\mathrm{TH}^{\cdot} \mathrm{An}^{\cdot+}\right) \mathrm{RP},(b)$ ${ }^{3}\left(\mathrm{SeH}^{\cdot} \mathrm{An}^{*+}\right)$ RP. (c) Comparison of the magnetic field dependence of geminate recombination rate constant $k_{\text {rec }}(B)$ for the two kinds of RPs: $O^{3}\left(\mathrm{TH}^{*} \mathrm{An}^{++}\right)$, ${ }^{3}\left(\mathrm{SeH}^{\cdot} \mathrm{An}^{\cdot+}\right)$.

ground state $\mathrm{TH}^{+}, 610 \mathrm{~nm}$, in the same kind of reverse micelles. Two absorption maxima of the selenine radical ${ }^{2} \mathrm{SeH}^{*}$ were observed at short wavelength, 410 and $425 \mathrm{~nm}$, which is consistent with the absorption spectrum of ${ }^{2} \mathrm{TH}^{\cdot}$ [21].

Transient signals representing the magnetic field dependent RP decay for ${ }^{3}\left(\mathrm{SeH}^{*}\right.$ $\mathrm{An}^{\cdot+}$ ) are shown in figure $1(\mathrm{~b})$. They have been obtained under the same conditions as the curves shown for ${ }^{3}\left(\mathrm{TH}^{-} \mathrm{An}^{-+}\right)$in figure $1(\mathrm{a})$. It is apparent that the radical decay is faster in the case of selenine than of thionine even at zero magnetic field, and that the effect of an external magnetic field is weaker in the selenine case. Figure 


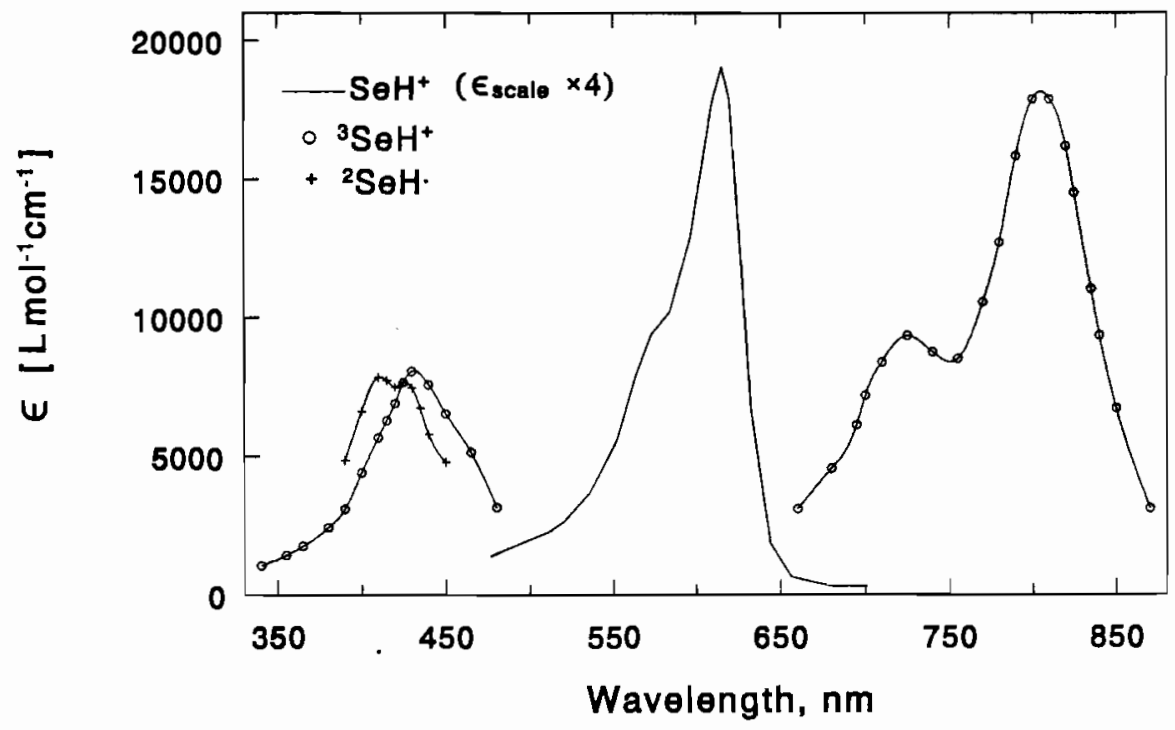

Figure 2. Absorption spectra of ground state selenine $\left(\mathrm{SeH}^{+}\right)$, selenine triplet $\left({ }^{3} \mathrm{SeH}^{+}\right)$and semireduced selenine radical $\left({ }^{2} \mathrm{SeH}\right)$. The radical was produced by triplet quenching with $0.05 \mathrm{M}$ aniline in CDBA/benzene reverse micellar system $(w=15)$ at $25^{\circ} \mathrm{C}$. Absolute $\varepsilon$-values of ${ }^{3} \mathrm{SeH}^{+}$and ${ }^{2} \mathrm{SeH}^{*}$ are only approximate. The observed transient absorption values have been normalized assuming saturation for the triplet spectrum and $100 \%$ triplet to radical conversion for the radical spectrum. For $\mathrm{SeH}^{+}$the $\varepsilon$-scale has to be multiplied by a factor of four.

1 (c) illustrates how the evaluated $\mathrm{RP}$ recombination rate constant $k_{\text {rec }}$ depends on the magnetic field $(B)$ for both ${ }^{3}\left(\mathrm{TH}^{*} \mathrm{An}^{*+}\right)$ and ${ }^{3}\left(\mathrm{SeH}^{*} \mathrm{An}^{*+}\right)$ RPs. In both cases, $k_{\text {rec }}$ seems to be saturated at fields of several kilogauss. The values of the recombination constants for the RPs with ${ }^{2} \mathrm{SeH}^{\cdot}\left({ }^{2} \mathrm{TH}^{*}\right)$ are: $k_{\mathrm{rec}}^{\mathrm{ZF}}=5.4 \times 10^{6} \mathrm{~s}^{-1}\left(2.7 \times 10^{6} \mathrm{~s}^{-1}\right)$ at zero field and $k_{\mathrm{rec}}^{\mathrm{HF}}=3.6 \times 10^{6} \mathrm{~s}^{-1}\left(1.08 \times 10^{6} \mathrm{~s}^{-1}\right)$ at $3.3 \mathrm{~T}$.

Considering the various contributions to $k_{\mathrm{rec}}^{\mathrm{ZF}}$ appearing in equation (1), the difference between ${ }^{2} \mathrm{SeH}^{\cdot}$ and ${ }^{2} \mathrm{TH}^{\cdot}$ could be due either to changes of $k_{S}$, or of $k_{T}$, or of both. A specific heavy atom effect should show up in $k_{r}$. Note that, as has been pointed out above, a possible heavy atom effect on the $T / S$ spin equilibration rate will not affect $k_{\mathrm{rec}}^{\mathrm{ZF}}$, since in zero field the spin equilibrium process is not rate determining in the present systems. If the introduction of a heavy atom leads to a sizeable increase in $k_{T}$ this should be reflected in a still stronger relative increase of the high field value of $k_{\text {rec }}$, as is in fact observed. However, we have to exclude the possibility that the high-field value is dominated by heavy-atom induced relaxation, i.e. by a heavy atom effect on $k_{r}$. Let us assume for the moment that $k_{r}$ is negligibly slow at high field. Then from equations (1) and (2) and the limiting values of $k_{\text {rec }}$ given above we can calculate $k_{S}$ and $k_{T}$ yielding for $\mathrm{SeH}^{*}$ (and $\mathrm{TH}^{*}$, respectively) $k_{S}=10.9 \times 10^{6} \mathrm{~s}^{-1}\left(7.6 \times 10^{6} \mathrm{~s}^{-1}\right)$ and $k_{T}=3.62 \times 10^{6} \mathrm{~s}^{-1}\left(1.1 \times 10^{6} \mathrm{~s}^{-1}\right)$, i.e. a 3.5 fold increase in $k_{T}$ as a clear manifestation of the heavy atom affect, but only a change by a factor of about 1.5 in $k_{s}$ reflecting, as expected, a greater similarity in the spin-allowed reactivity of both dyes. Thus, the result of this analysis is in favour of the hypothesis that heavy-atom substitution does in fact induce a $k_{T}$ effect and not a $k_{r}$ effect. It must be emphasized, though, that the strength of this argument relies on the supposition that $k_{s}$ should be about the same for $\mathrm{TH}^{*}$ and $\mathrm{SeH}^{*}$. This assumption is reasonable since $k_{s}$ may be considered as diffusion controlled (see [12]) 

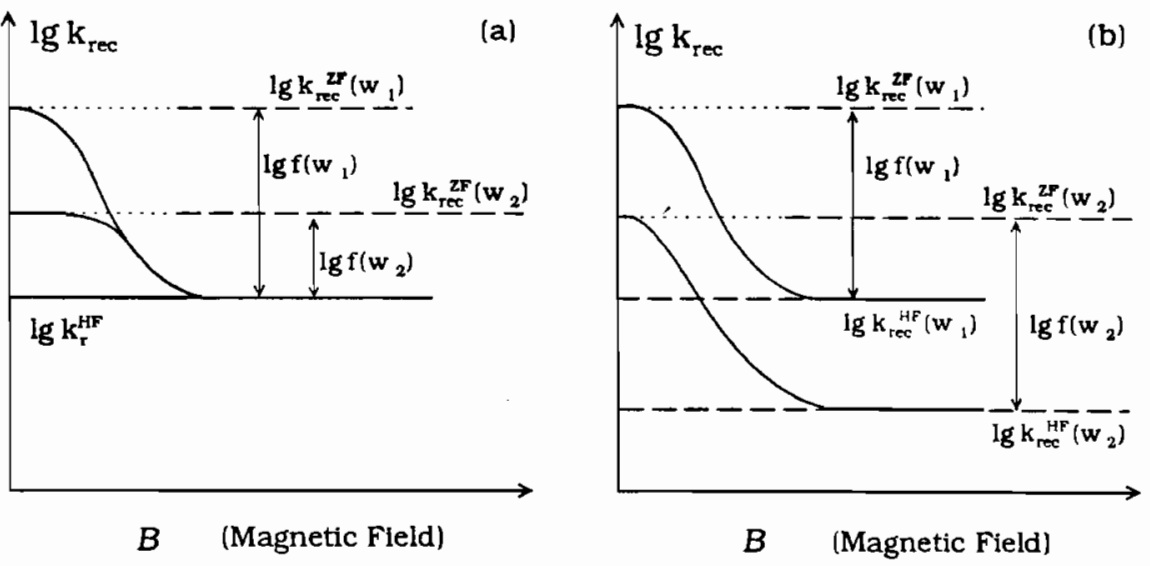

Figure 3. Schematic representation of the magnetic field dependence of RP recombination rate constant $k_{\text {rec }}$ in micellar supercages of two different sizes $\left(w_{1}<w_{2}\right)$. (a) case of $w$-independent high-field values; determined by a magnetic-field independent intraradical relaxation process; (b) case of $w$-dependent high-field values.

and $\mathrm{TH}^{\circ}$ and $\mathrm{SeH}^{\circ}$ are expected tp prefer the same micellar microenvironment and have about the same diffusion coefficient. However, we can provide additional experimental evidence in favour of the $k_{T}$-hypothesis.

As noted above, SOC effects on $k_{T}$ are expected to be associated with the situation of RPs in contact, whereas SOC effects on $k_{r}$ as resulting from sri or gta, should be independent of the RP distance. Since the relative contributions of processes with the $\mathbf{R} \mathbf{P}$ in contact as compared to those with separate radicals are modified when changing the radius of the nanoscopic cage, two different limiting cases may be anticipated (see figure 3):

(a) If the recombination kinetics at high field is limited by a field-independent intra-radical relaxation process, its rate constant $k_{\mathrm{rec}}^{\mathrm{HF}}$ should be rather independent of the micellar size (provided that other parameters like the microviscosity are not changing with it). On the other hand, the recombination rate constant in zero field $k_{\text {rec }}^{\text {zF }}$ which is completely controlled by the frequency of encounters, will strongly decrease with increasing radius (in our experiments accomplished by an increase of the water/surfactant ratio $w$ ). Therefore the 'saturation' ratio $f \equiv k_{\mathrm{rec}}^{\mathrm{ZF}} / k_{\mathrm{rec}}^{\mathrm{HF}}$ of the magnetic field effect will strongly decrease with increasing $w$.

(b) If the recombination kinetics at high field is limited by $k_{T}, k_{\mathrm{rec}}^{\mathrm{HF}}$ is limited by inter-radical ISC processes which are expected to exhibit the same wdependence as the diffusion controlled processes determining $k_{\mathrm{rec}}^{\mathrm{ZF}}$. Thus, in this case, the saturation ratio $f$ is assumed to be fairly independent of $w$.

In order to decide which of these cases applies, we investigated the RP kinetics of ${ }^{3}\left(\mathrm{SeH}^{\cdot} \mathrm{An}^{\cdot+}\right)$ RPs in reverse micelles of different size $(w=8 \ldots 30$ corresponding to a range of radii of $17 \AA \ldots 52 \AA$ ). The characteristic parameters evaluated are compared in table 1 . Through the increase of the micellar radius the intramicellar RP kinetics are strongly slowed down. The $w$-dependence of the individual rate constants $k_{\text {esc }}$ and $k_{\text {rec }}^{\mathrm{ZF}}$ are displayed in figure 4 . They exhibit an approximate $1 / r^{3}$ $\left(r^{-2 \cdot 6}\right)$ dependence in the case of $k_{\text {rec }}$ and an $r^{-1}$ dependence in the case of $k_{\text {esc }}$. These findings are quite similar to what we observed and discussed in the case of 
Table 1. Evaluated parameters characterizing the micelle-size and magnetic-field dependence of ${ }^{3}\left(\mathrm{SeH}^{*} \mathrm{An}^{*+}\right) \mathrm{RP}$ recombination in water/CDBA/benzene reverse micelles with $[\mathrm{CDBA}]=0.04 \mathrm{M},[\mathrm{An}]=0.05 \mathrm{M}$ and $\left[\mathrm{SeH}^{+}\right]=5 \times 10^{-6} \mathrm{M} \cdot$

\begin{tabular}{lccccccc}
\hline$w$ & $k_{\text {rec }}^{0 \mathrm{~T}} /\left(10^{6} \mathrm{~s}^{-1}\right)$ & $k_{\mathrm{rec}}^{3.3 \mathrm{~T}} /\left(10^{6} \mathrm{~s}^{-1}\right)$ & $f^{(a)}$ & $B_{1 / 2}{ }^{(b)} /(\mathrm{G})$ & $\overline{k_{\text {esc }}} /\left(10^{6} \mathrm{~s}^{-1}\right)$ & $\Phi_{\text {esc }}^{0 \mathrm{~T}}$ & $\Phi_{\text {esc }}^{3.3 \mathrm{~T}}$ \\
\hline 8 & 13.6 & 10.46 & 1.3 & 129 & 3.23 & 0.195 & 0.23 \\
11.5 & 8.31 & 6.04 & 1.4 & 107 & 2.25 & 0.21 & 0.28 \\
15 & 5.44 & 3.63 & 1.5 & 113 & 1.76 & 0.25 & 0.33 \\
22.5 & 1.76 & 1.29 & 1.4 & 114 & 1.02 & 0.38 & 0.45 \\
30 & 0.91 & 0.64 & 1.4 & 152 & 0.84 & 0.51 & 0.56 \\
\hline
\end{tabular}

(a) $f \equiv k_{\mathrm{rec}}^{0 \mathrm{~T}} / k_{\mathrm{rec}}^{3 \cdot 3 \mathrm{~T}}$.

${ }^{(b)}$ Magnetic field at which $k_{\mathrm{rec}}$ equals the mean value of $k_{\mathrm{rec}}^{0 \mathrm{~T}}$ and $k_{\mathrm{rec}}^{3 \cdot 3 \mathrm{~T}}$.
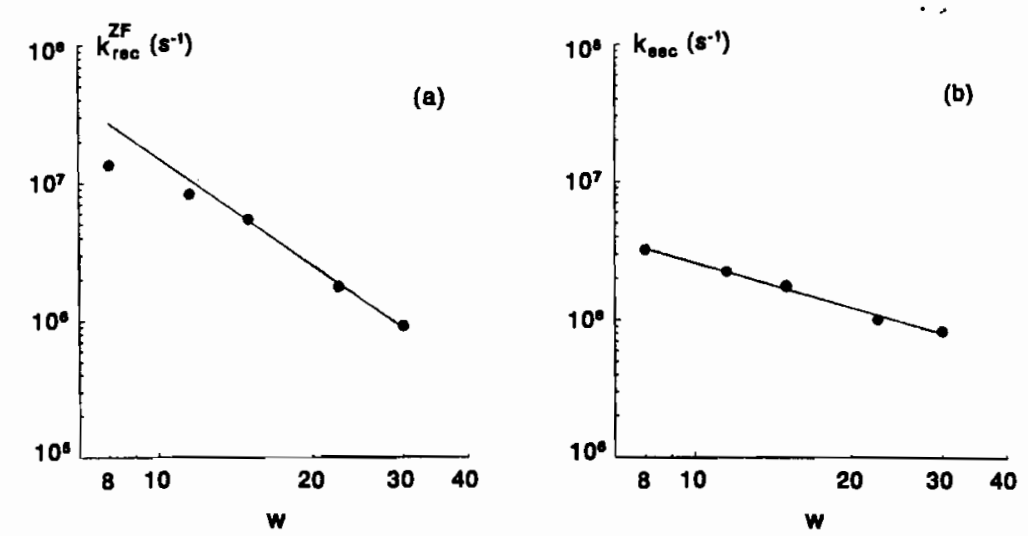

Figure 4. The $w$-dependence of ${ }^{3}\left(\mathrm{SeH}^{\cdot} \mathrm{An}^{\cdot+}\right)$ RP kinetics at zero magnetic field. (a) rate constant of recombination $k_{\mathrm{rec}}$ and $(b)$ rate constant of escape $k_{\mathrm{esc}}$.

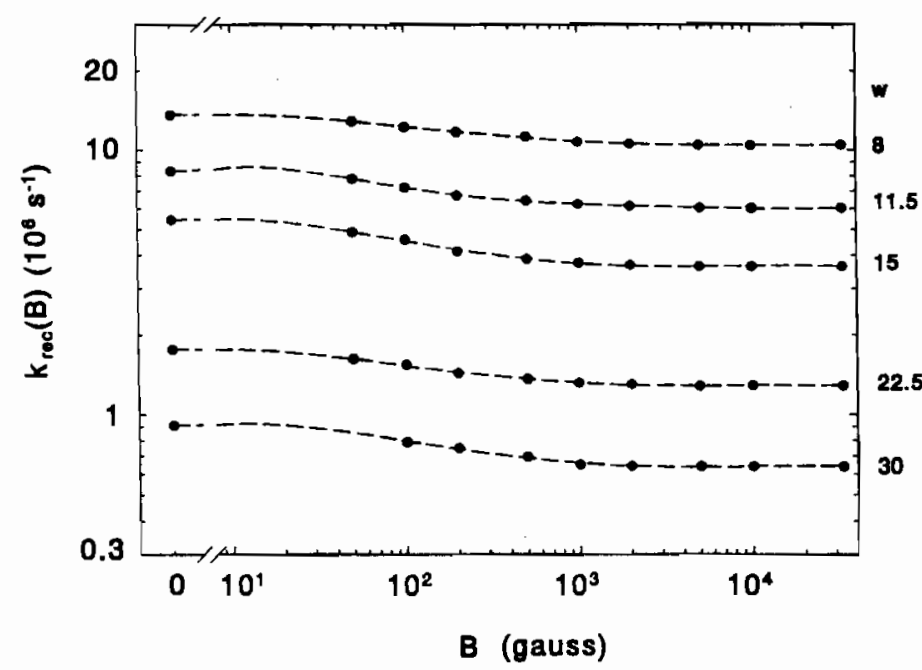

Figure 5. Magnetic-field dependence of the rate constant $k_{\text {rec }}$ of geminate recombination of ${ }^{3}\left(\mathrm{SeH}^{*} \mathrm{An}^{*+}\right) \mathrm{RP}$ in water/CDBA/benzene reverse micelles of variable size. 
${ }^{3}\left(\mathrm{TH}^{\bullet} \mathrm{An}^{\cdot+}\right)$ RPs [26]. In Figure 5 we show the magnetic field dependence of $k_{\text {rec }}$. Comparing the magnetic field dependence exhibited by the RPs under a variationof the micellar size (figure 5) with the schematic cases in figure 3 , it is clearly apparent that the observed behaviour corresponds to case $(b)$ i.e. the saturation ratio $f$ is constant (see table 1). Thus we can conclude that the increase of SOC resulting from substitution of $\mathrm{S}$ by $\mathrm{Se}$ is exclusively manifested in the increase of $k_{T}$ (inter-radical ISC) while SOC dependent intra-radical contributions to $k_{r}$ are not detectable (for a discussion of the $w$-dependence of $k_{r}$ see section 4.3 ).

\subsection{Effect of a heavy atom in the oxidized radical: $p$-Cl-aniline cation radical}

As has been reported in [21] the magnetic field effect observed with ${ }^{3}\left(\mathrm{TH}^{*} X\right.$ $\mathrm{An}^{{ }^{+}}$) RPs in reverse microemulsion nanodroplets is systematically reduced with increasing nuclear charge in the series of para-substituted aniline cations $\mathrm{p}-X-\mathrm{An}^{*+}$. With $\mathrm{p}-\mathrm{Cl}-\mathrm{An}^{*+}$ in microemulsions of $w=15$ the high-field MFE corresponds to a decrease in $k_{\text {rec }}$ by $54 \%(f=2 \cdot 2)$ as compared to $66 \%(f=2 \cdot 9)$ with $\mathrm{An}^{\cdot+}$. Although, in microemulsions with $w=15$, the heavy atom effect due to the Cl-substituent is small, this case seems to be most suitable to test the hypothesis of a $k_{T}$-type heavy-atom effect against the hypothesis of a $k_{r}$-type heavy-atom effect since here the $w$-dependence of $f$ should become most clearly apparent. If the heavy-atom effect of the $\mathrm{Cl}$ substituent were due to an enhancement of field-independent intra-radical contributions to $k_{r}\left(k_{r}^{\mathrm{HAE}}\right)$ the $f$-values given above together with the value of the zero-field rate constant $k_{\mathrm{rec}}^{\mathrm{ZF}}=3.3 \times 10^{6} \mathrm{~s}^{-1}$ would imply that $k_{r}^{\mathrm{HAE}} \approx(2.9-2.2) \times$ $3.3 \times 10^{6} \mathrm{~s}^{-1}=2.3 \times 10^{6} \mathrm{~s}^{-1}$ for $\mathrm{p}-\mathrm{Cl}-\mathrm{An}^{-+}$. This value is expected to change only little with the size of the microemulsion nanodroplets. In figure 6 we show the field dependence of $k_{\mathrm{rec}}$ for $w=8,15,22$. While under the variation of $w, k_{\mathrm{rec}}^{\mathrm{ZF}}$ covers a range from $7.1 \times 10^{6} \mathrm{~s}^{-1}$ to $1.8 \times 10^{6} \mathrm{~s}^{-1}$ it is obvious that a variation of $w$ has negligible effect on the relative field dependence $R\left(k_{\mathrm{rec}}\right)$ of $k_{\text {rec }}$ and, in particular, on the saturation value $f$. If it were for changes in $k_{r}^{\mathrm{HAE}}$ to be responsible for the

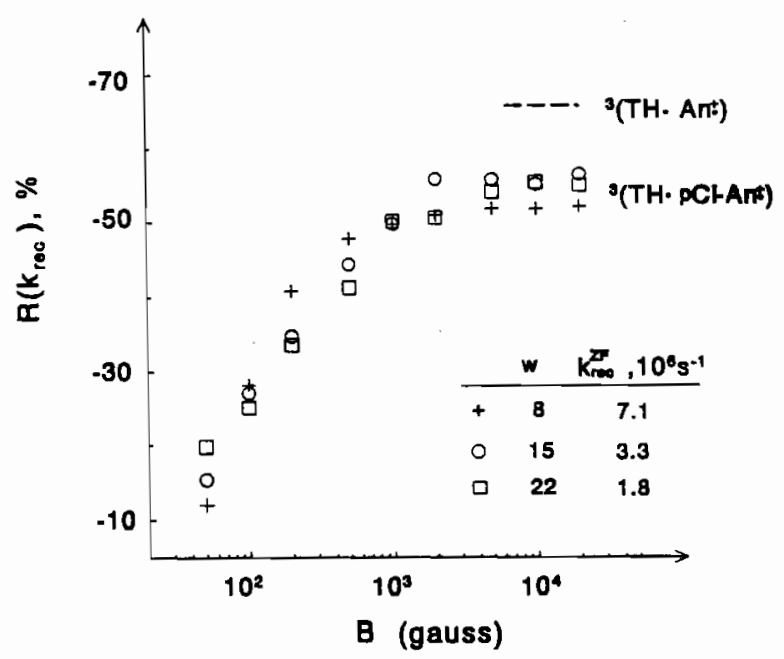

Figure 6. Relative MFE $\left.R\left(k_{\mathrm{rec}}\right) \equiv\left[\left(k_{\mathrm{rec}}\left(B_{0}\right)-k_{\mathrm{rec}}^{\mathrm{ZF}}\right) / k_{\mathrm{rec}}^{\mathrm{ZF}}\right)\right]$ on the rate constant of geminate recombination of ${ }^{3}\left(\mathrm{TH}^{*} \mathrm{p}-\mathrm{Cl}-\mathrm{An}^{+}\right) \mathrm{RPs}$ in water/CDBA/benzene microemulsions for various radii $(\propto w)$. The dashed line represents the high-field value of the MFE measured with ${ }^{3}\left(\mathrm{TH}^{*} \mathrm{An}^{*+}\right)$ RPs at $w=15$. 
heavy-atom effect, the $w$-independence of this quantity would lead us to expect that $f=1.5$ for $w=8$ and $f \approx 1$ for $w=22$ (note that for $w=22$ the observed $k_{\mathrm{rec}}^{\mathrm{zF}}$ is already smaller than the hypothetical $k_{r}^{\mathrm{HAE}}$-value!). However, $f$ is found to be $2 \cdot 2$ throughout. Thus again, based on direct experimental evidence, we arrive at the conclusion that the increase of SOC by intra-radical heavy atom substitution leads to an enhancement of $k_{T}$, a quantity related to inter-radical SOC. While this has now been unequivocally established, it may be of interest to obtain a theoretical estimate of the actual heavy-atom contributions to intra-radical spin relaxation.

\subsection{Theoretical estimation of heavy-atom induced contributions to $\mathrm{k}_{\mathrm{r}}$}

Intraradical $T_{1}$-processes contribute to $k_{r}$ of the RP according to the relation [3]

$$
k_{r}=\frac{1}{2 T_{1(1)}}+\frac{1}{2 T_{1(2)}},
$$

where $T_{1(i)}$ is the electron spin resonance (ESR) $T_{1}$ time of radical $i$.

We will now estimate the change in $k_{r}$ owing to the gta and the sri mechanisms in the radical carrying the heavy atom $\mathrm{Se}$ or $\mathrm{Cl}$, respectively. For the gta-mechanism the pertinent relations are [3]

with

$$
\left(\frac{1}{T_{l}}\right)_{\mathrm{gta}}=\frac{1}{20} \overline{\Delta g^{2}} \frac{\omega_{0}^{2} \tau_{\mathrm{c}}}{1+\omega_{0}^{2} \tau_{\mathrm{c}}^{2}}
$$

In the high field (HF) limit:

$$
\omega_{0}=2 \beta \hbar^{-1} B_{0}
$$

For the sri mechanism one has:

$$
\left(\frac{1}{T_{1}}\right)_{\mathrm{gta}}^{\mathrm{HF}}=\frac{1}{20} \frac{\overline{\Delta g^{2}}}{\tau_{\mathrm{c}}}
$$

$$
\left(\frac{1}{T_{1}}\right)_{\mathrm{sri}}=\frac{\overline{\delta \mathbf{g}^{2}}}{9 \tau_{\mathrm{c}}}
$$

where the $\mathbf{g}$ tensor anisotropy and its deviation from the free electron value are defined as:

$$
\begin{aligned}
& \overline{\Delta \mathbf{g}^{2}} \equiv \sum g_{i i}^{2}-\frac{1}{3}\left(\sum g_{i i}\right)^{2} \\
& \overline{\delta \mathbf{g}^{2}} \equiv \sum\left(g_{i i}-2 \cdot 0023\right)^{2}
\end{aligned}
$$

and the reorientational correlation time is given by:

$$
\tau_{\mathrm{c}}=\frac{4 \pi \eta r^{3}}{3 k T}=10^{-12} \frac{\eta}{c P} \frac{r^{3}}{\AA^{3}} \mathrm{~s},
$$

with $\eta$ the dynamic solvent viscosity and $r$ the hydrodynamic radius of the radical. For the g-tensor components of the $\mathrm{p}$-Cl-aniline cation radical we use one half of the values given in [28] for the 5-chlorouracil radical, where the spin density at the halogen is approximately half or less of that of the corresponding p-halogenaniline radical. (The latter conclusion is derived from a comparison of the experimental hyperfine coupling constants of the 5-fluorouracil radical (42 G [28]) and the $\mathrm{N}, \mathrm{N}$-dimethyl-p-fluoroaniline radical (20.8 G [29]).) The upper limits of the g-tensor 
Table 2. Estimated values of spin relaxation rate constants due to gta and sri mechanism.

\begin{tabular}{|c|c|c|c|c|c|}
\hline \multirow[b]{2}{*}{$w$} & \multirow[b]{2}{*}{$\eta^{(a)}$} & \multicolumn{2}{|c|}{$\mathrm{p}-\mathrm{Cl}-\mathrm{An}^{-+}$} & \multicolumn{2}{|c|}{$\mathrm{SeH}^{\cdot}$} \\
\hline & & $k_{r}^{\text {gta }} /\left(\mathrm{s}^{-1}\right)$ & $k_{r}^{3 r i} /\left(s^{-1}\right)$ & $k_{r}^{\mathrm{gta}} /\left(\mathrm{s}^{-1}\right)$ & $k_{r}^{s r i} /\left(s^{-1}\right)$ \\
\hline 8 & 6 & $2.6 \times 10^{3}$ & $1.5 \times 10^{4}$ & $8 \cdot 3 \times 10^{3}$ & $5.5 \times 10^{4}$ \\
\hline $11 \cdot 5$ & 6 & & & $8.3 \times 10^{3}$ & $5.5 \times 10^{4}$ \\
\hline 15 & $4 \cdot 8$ & $3 \cdot 3 \times 10^{3}$ & $1.9 \times 10^{4}$ & $1.0 \times 10^{4}$ & $69 \times 10^{4}$ \\
\hline $22 \cdot 5$ & $3 \cdot 2$ & $5.0 \times 10^{3}$ & $2.9 \times 10^{4}$ & $1.6 \times 10^{4}$ & $1.04 \times 10^{5}$ \\
\hline 30 & $2 \cdot 5$ & & & $2.0 \times 10^{4}$ & $1.34 \times 10^{5}$ \\
\hline
\end{tabular}

(a) Dynamic solvent viscosity in $\mathrm{cP}$ units, determined by ESR spin probe measurements according to equation (14) [24].

values thus estimated for the p-Cl-aniline radical are $\mathbf{g}_{x x}=2.00615, g_{y y}=2.00565$ and $g_{z z}=2.00205$, from which one obtains $\overline{\Delta \mathbf{g}^{2}}=10^{-5}$, and $\overline{\delta \mathbf{g}^{2}}=2.6 \times 10^{-5}$. For $\mathrm{SeH}^{*}$ only the isotropically averaged value of $\overline{\mathbf{g}}=2.0083$ in solution is known [30]. Assuming that the g-tensor is of approximately axial symmetry and that only $\mathbf{g}_{\perp}$ deviates from the free electron value we obtain $\overline{\Delta \mathbf{g}^{2}}=5.4 \times 10^{-5}$ and $\overline{\delta \mathbf{g}^{2}}=$ $1.62 \times 10^{-4}$ for this radical. Effective microviscosity values experienced by hydrophilic solutes in the microemulsion droplets have been obtained by the ESR spin probe technique using protonated tempamine as a probe [24]. These values (see table 2) and effective hydrodynamic radii of $2.5 \AA$ for p-Cl- $\mathrm{An}^{+}$and $3 \AA$ for $\mathrm{SeH}^{*}$ allow us to estimate the $k_{r}$-contributions $k_{r}^{\text {gta }}$ and $k_{r}^{\text {sri }}$ listed in table 2 . The values, being by about a factor of three higher for $\mathrm{SeH}^{*}$ than for $\mathrm{p}-\mathrm{Cl}-\mathrm{An}^{+}$, are significantly smaller than the $k_{\mathrm{rec}}$ values observed in the high field saturation limits. It should be noted that our present value for $\mathrm{p}-\mathrm{Cl}-\mathrm{An}^{\cdot+}$ has been corrected against a larger one estimated in our earlier work [21], the discrepancy being mainly due to the factor of $\frac{1}{2}$ relating $1 / T$ and $k_{r}$ (see equation (7)) and the employed microviscosities, not exactly known previously.

From the results of this section we may conclude, that the theoretical estimates, too, corroborate our interpretation that the heavy-atom effect on the magnetic field dependent RP kinetics is almost exclusively due to a $k_{T}$ effect. It should be added here that for the radicals $\mathrm{An}^{\circ+}$ and $\mathrm{TH}^{*}$ the corresponding contributions to $k_{r}$ must be even smaller. Therefore, in the case of this radical pair, too, the relaxation mechanism fails to account for the high-field value $k_{\mathrm{rec}}^{\mathrm{HF}}$ of $k_{\mathrm{rec}}$ which is in fact about $\frac{1}{3}$ of $k_{\mathrm{rec}}^{\mathrm{ZF}}$, independent of $w$. This justifies the assumption made in a previous paper [12] that for the $\left(\mathrm{TH}^{*} \mathrm{An}^{\cdot+}\right) \mathrm{RP}$ the recombination kinetics in high field is determined by the $k_{T}$ process.

\section{Conclusions}

Using the combined effects of heavy atom substitution and variation of nanoreactor size on the magnetic field dependence of micellar radical pair kinetics, we have shown that direct formation of singlet recombination products can ensue during encounters of triplet radical pairs. The rate constants of this 'spin-forbidden' recombination can be estimated from the ratio $f$ of zero-field to high-field value of 
the recombination rate constant.

$$
\begin{gathered}
f \equiv \frac{k_{\mathrm{rec}}^{\mathrm{ZF}}}{k_{\mathrm{rec}}^{\mathrm{HF}}}=\frac{\frac{1}{4} k_{S}+\frac{3}{4} k_{T}}{k_{T}}=\frac{1}{4} \frac{k_{S}}{k_{T}}+\frac{3}{4}, \\
\frac{k_{T}}{k_{S}}=(4 f-3)^{-1} .
\end{gathered}
$$

Thus we obtain $k_{T} / k_{S} \approx 0.38$ for the $\left(\mathrm{SeH}^{*} / \mathrm{An}^{\cdot+}\right) \mathrm{RP}, 0.17$ for $\left(\mathrm{TH}^{*} / \mathrm{p}-\mathrm{Cl}-\mathrm{An}^{\circ+}\right)$, and 0.12 for $\left(\mathrm{TH}^{*} / \mathrm{An}^{*+}\right)$. Assuming that the recombination efficiency of a S-RP is unity, the values of $k_{T} / k_{S}$ can be interpreted as probabilities of T-RP recombination in one encounter or, put into a different perspective, the compliment of its value to 1 should represent the probability of T-RP escape from the 'normal' solvent cage (as different from the micellar supercage) surrounding the $\mathrm{RP}$ while in contact. The corresponding 'normal' cage escape efficiencies would be 0.62 for $\left(\mathrm{SeH}^{*} / \mathrm{An}^{*+}\right), 0.83$ for $\left(\mathrm{TH}^{*} / \mathrm{p}\right.$ $\left.\mathrm{Cl}-\mathrm{An}^{*+}\right)$, and 0.88 for $\left(\mathrm{TH}^{*} / \mathrm{An}^{\circ+}\right)$. In fact, these values range well in the region observed for cage-escape efficiencies in homogeneous solution of comparable microviscosity with T-RPs of similar SOC-strength involving thiopyronine semiquinone and halogen-anilines [31]. In that and related work [15,32] we have provided evidence that there is an efficient, SOC-induced reaction leading from triplet-exciplex type contact T-RPs to singlet ground state recombination products. Quantum mechanically these processes are accounted for by an electronic coupling matrix element associated with the transfer of an electron between the two radicals, i.e. directly connected with the backward electron transfer process.

Financial support by the Deutsche Forschungsgemeinschaft and the Fonds der Chemischen Industrie is gratefully acknowledged.

\section{References}

[1] Salikhov, K. M., Molin, Yu. N., Sagdeev, R. Z., and Buchachenko, A. L., 1984, Spin Polarization and Magnetic Effects in Radical Reactions (Amsterdam).

[2] Steiner, U. E., and Ulrich, T., 1989, Chem. Rev., 89, 51.

[3] Steiner, U. E., and Wolff, H.-J., 1991, Photochemistry and Photophysics, Vol. 4, edited by J. F. Rabek (Boca Raton: CRC Press), p. 1.

[4] Khudyakov, I. V., Serebrennikov, Y. A., and Turro, N. J., 1993, Chem. Rev., 93, 537.

[5] Kaptein, R., and Oosterhoff, L. J., 1969, Chem. Phys. Lett., 4, 195, 214.

[6] Closs, G. L., and Trifunac, A. D., 1970, J. Am. Chem. Soc., 92, 2183.

[7] Turro, N. J., 1983, Proc. natl. Acad. Sci. U.S.A., 80, 609.

[8] Buchachenko, A. L., 1984, Prog. React. Kinet., 13, 163.

[9] Turro, N. J., and Weed, G. C., 1983, J. Am. Chem. Soc., 105, 1861.

[10] Hayashi, H., and NagakuRa, S., 1984, Bull. chem. Soc Japan., 57, 322.

[11] Lüders, K., and Salikhov, K. M., 1987, Chem. Phys., 117, 113.

[12] Steiner, U. E., and Wu, J. Q., 1992, Chem. Phys., 162, 53.

[13] Steiner, U. E., 1980, Chem. Phys. Lett., 74, 108.

[14] Steiner, U. E., 1981, Ber. Bunsenges. phys. Chem., 85, 228.

[15] Ulrich, T., Steiner, U. E., and Föll, R. E., 1983, J. phys. Chem., 87, 1873.

[16] Closs, G. L., 1979, Chemically Induced Magnetic Polarization, edited by L. T. Muus, P. W. Atkins and K. A. McLauchlan (Dordrecht: Reidel), p. 225.

[17] Doubleday Jr, J., 1979, Chem. Phys. Lett., 64, 71; 1981, Ibid. 79, 375.

[18] Minaev, B. F., and Lunell, S., 1993, Z. Phys. Chem., 182, 263.

[19] Doubleday, C. J. JR, Turro, N. J., and Wang, J. F., 1989, Accts. Chem. Res., 22, 199 . 
[20] Levin, P. P., and Kuzmin, V. A., 1990, Chem. Phys. Lett., 165, 302.

[21] Ulrich, T., Steiner, U. E., and SChlenkeR, W., 1986, Tetrahedron, 42, 6131.

[22] Schulten, K., and Wolynes, P. G., 1978, J. chem. Phys., 68, 3292.

[23] (a) Endriss, W., 1961, Doctoral Thesis, Universität Stuttgart; (b) Vogelmann, E., 1974, Doctoral Thesis, Universität Stuttgart.

[24] Wu, J. Q., 1993, Doctoral Thesis, Universität Konstanz.

[25] ULRICH, T., 1986, Doctoral Thesis, Universität Konstanz.

[26] Ulrich, T., and Steiner, U. E., 1984, Chem. Phys. Lett., 112, 365.

[27] Vogelmann, E., and Kramer, H. E. A., 1976, Photochem. Photobiol., 23, 383.

[28] Sevilla, M. D., Swarts, S., Riederer, H., and Hüttermann, J., 1984, J. Phys. Chem., 88, 1601.

[29] Latta, B. M., and Taft, R. W., 1967, J. Am. Chem. Soc., 89, 5172.

[30] SCHMIDT, H., private communication.

[31] Winter, G., and STEINER, U. E., 1980, Ber. Bunsenges. phys. Chem., 84, 1203.

[32] Steiner, U. E., and Winter, G., 1978, Chem. Phys. Lett., 55, 364. 